\title{
MEASURED AND SIMULATED DARK J-V CHARACTERISTICS OF a-Si:H SINGLE JUNCTION p-i-n SOLAR CELLS IRRADIATED WITH 40keV ELECTRONS ${ }^{1}$
}

Kenneth Lord and James R. Woodyard

Wayne State University, Detroit, Ml 48202

\begin{abstract}
The effect of $40 \mathrm{keV}$ electron irradiation on a-Si:H p-i-n single-junction solar cells was investigated using measured and simulated dark J-V characteristics. EPRI-AMPS and PC-1D simulators were explored for use in the studies. The EPRI-AMPS simulator was employed and simulator parameters selected to produce agreement with measured J-V characteristics. Three current mechanisms were evident in the measured dark J-V characteristics after electron irradiation, namely, injection, shunting and a term of the form $\mathrm{CV}^{\mathrm{m}}$. Using a single discrete defect state level at the center of the band gap, good agreement was achieved between measured and simulated J-V characteristics in the forward-bias voltage region where the dark current density was dominated by injection. The current mechanism of the form $\mathrm{CV}^{\mathrm{m}}$ was removed by annealing for two hours at $140^{\circ} \mathrm{C}$. Subsequent irradiation restored the $\mathrm{CV}^{\mathrm{m}}$ current mechanism and it was removed by a second anneal. Some evidence of the $C V^{m}$ term is present in device simulations with a higher level of discrete density of states located at the center of the bandgap.
\end{abstract}

\section{INTRODUCTION}

Thin-film a-Si:H solar cells are prime candidates for space applications because investigations show that they have good radiation resistance [1]. The fabrication technology may be automated and has the potential for solar arrays with low cost per watt. The a-Si:H-based solar cell technology permits deposition on ultra-thin flexible substrates, thus offering high specific power density. To take advantage of the high specific power density, cells must be flown without a cover glass, thereby requiring that the nude device structure have good radiation resistance. In order to predict the radiation resistance of solar cells in space environments, particle irradiations are generally carried out in the laboratory with monoenergetic particle beams at normal incidence. In space environments there is a wide energy distribution of particles with a range of incident angles. Hence, the fundamental radiation resistance mechanisms must be understood if laboratory measurements are to be used to predict solar cell performance in a variety of space environments.

The goal of our program is to acquire a detailed understanding of the effect of particle irradiation on thin-film solar cells. We have carried out studies on the effect of MeV proton irradiation on single and multi-junction a-Si:H cells; dark current mechanisms have been identified in both virgin and irradiated cells [2]. The proton irradiation studies have shown that dark $\mathrm{J}-\mathrm{V}$ characteristics are orders of magnitude more sensitive to radiation effects than light $\mathrm{J}-\mathrm{V}$ characteristics. Woodyard and Landis reviewed the published literature on electron irradiation studies on thinfilm devices [3]; materials and device structures have improved since the review was published. Cells are thinner and more efficient than those studied in earlier work. It is important to study and characterize the effect of particle irradiation on state-of-the-art cells if current technology is to be advanced. The AE8MAX model [4] predicts a tenyear integrated electron flux of $1.7 \mathrm{E} 16 \mathrm{~cm}^{-2}$ for electrons in the $50 \mathrm{keV}$ to $10 \mathrm{MeV}$ range incident on a surface in geosynchronous orbit. The model does not include flux from solar flares and fluxes are questionable at the lower electron energies. The flux of electrons in the model increases at lower energies. It is for this reason that our initial electron irradiation work was carried out with electron beam energies of $40 \mathrm{keV}$ and fluences of about $1 \mathrm{E} 17$ $\mathrm{cm}^{-2}$.

\footnotetext{
${ }^{1}$ This work was supported under NASA Grant NAG 3-2180.
} 
A single-junction a-Si:H p-i-n device structure was selected for this work. Single-junction $\mathrm{p}$-i-n cells without back reflectors, as opposed to multi-junction cells with back reflectors, were studied for two reasons. The singlejunction $\mathrm{p}-\mathrm{i}-\mathrm{n}$ device structure is the basic structure employed in multi-junction a-Si:H solar cells. An understanding of the single-junction a-Si: $\mathrm{H} \mathrm{p}-\mathrm{i}-\mathrm{n}$ device structure may make it possible to predict the performance of multi-junction device structures. Additionally, the analysis of measured dark $\mathrm{J}-\mathrm{V}$ characteristics of a singlejunction $\mathrm{p}$-i-n device structure is considerably easier than those of multi-junction devices structures.

The study of the fundamental radiation resistance mechanisms in devices is complex. It is for this reason that both $\mathrm{J}-\mathrm{V}$ measurements of irradiated cells and device simulations of $\mathrm{J}-\mathrm{V}$ characteristics were employed in this work. In order to gain experience with available device simulators in calculating dark J-V characteristics, two simulators were used to study a two-sided step $p-n$ junction. The free electron densities, electric field and dark J$\checkmark$ characteristics of a two-sided step p-n junction can be calculated using an analytical model [5]. The simulators selected were EPRI-AMPS [6] and PC-1D [7]. Parameters were selected for each simulator to produce identical electron densities, electric field and dark J-V characteristics. The premise for this approach was, if two simulators developed independently and employing different algorithms could produce the same p-n junction characteristics, and the characteristics agree with an analytical model, then simulations of $p-i-n$ structures, which cannot be modeled analytically, may have some validity. Agreement between the simulators and analytical would also insure that we had an adequate understanding of the simulators to use them effectively.

Following irradiations with $40 \mathrm{keV}$ electrons, dark J-V characteristics were measured. Simulations of dark J-V characteristics of $\mathrm{p}-\mathrm{i}-\mathrm{n}$ solar cells were carried out and parameters selected to fit the simulations to the measurements. The parameters that resulted in good fits to the measured J-V characteristics in the various forward-bias ranges were used to develop an understanding of current mechanisms in both virgin and irradiated a-Si:H p-i-n solar cells.

\section{p-n JUNCTION SIMULATIONS}

A p-n junction device structure with Si material parameters was investigated with both PC-1D and EPRI-AMPS simulators and the results compared with each other and an analytical model for a two-sided $p-n$ step junction. The p-type and n-type layers were each $5000 \mu \mathrm{m}$ thick. A doping level of $1 \mathrm{E} 18 \mathrm{~cm}^{-3}$ was used for both acceptors in the p-layer and donors in the n-layer. For simplicity, a mobility of $1000 \mathrm{~cm}^{2} \mathrm{~V}^{-1} \mathrm{~s}^{-1}$ was chosen for both electrons and holes. In working with the simulators, we learned there are two major differences between the simulators. First, PC-1D is a transient simulator, and EPRI-AMPS is a steady-state simulator. Therefore, stepping times for sweeping device voltages must be considered when using the PC-1D simulator. Secondly, the PC-1D simulator uses a lifetime model and the EPRI-AMPS simulator employs an electronic density of states, DOS, model. In order to calculate free electron carrier densities from lifetimes with the EPRI-AMPS simulator, a lifetime subroutine is provided by the developer for calculating a DOS from carrier lifetimes. The PC-1D and EPRI-AMPS simulators produced vastly different free electron densities with the lifetime approach. It was concluded that the EPRI-AMPS simulator does not produce correct free electron densities when the lifetime approach is employed. The next approach was to use the DOS feature of EPRI-AMPS and to calculate a lifetime using a model that relates the DOS to a lifetime that could be used in the PC-1D simulator.

Figure 1 shows the simulated and calculated free electron concentration in the $p$-n junction under a forward bias of $0.5 \mathrm{~V}$. The front of the device is located at a positon of $0 \mu \mathrm{m}$ and the metallurgical junction at 5,000 $\mu \mathrm{m}$. The front $p$-type layer is within positions of 0 to $5,000 \mu \mathrm{m}$ and the rear $n$-type layer is within positions of 5,000 to $10,000 \mu \mathrm{m}$. The front and rear contacts in the simulators are located at 0 and $10,000 \mu \mathrm{m}$, respectively. Electrons are minority carriers in the $p$-type layer and the free carrier densities are about $1 \mathrm{E}^{2} \mathrm{~cm}^{-3}$ near the front contact of the device. Electrons are majority carriers in the n-type layer and have free carrier densities of about $1 \mathrm{E} 18 \mathrm{~cm}^{-3}$ near the back contact. Figure 1 shows that both PC-1D and EPRI-AMPS simulators produce a diffusion electron current density due to electron injection at the metallurgical junction. Electron injection is evidenced by the exponential decrease in the free electron carrier densities from the metallurgical junction forward into the p-type layer. The PC-1D simulator free electron density versus position was calculated using a Shockley Read Hall, $\mathrm{SRH}$, recombination lifetime of $10 \mu \mathrm{s}$. The band-to-band recombination coefficient was set to an unrealistically low number for simplicity so that the recombination is entirely dominated by SRH recombination. The EPRIAMPS simulator free electron density versus position shown in Figure 1 was calculated with a DOS distribution. The DOS distribution used was constant both with respect to position in the device as well as with respect to 


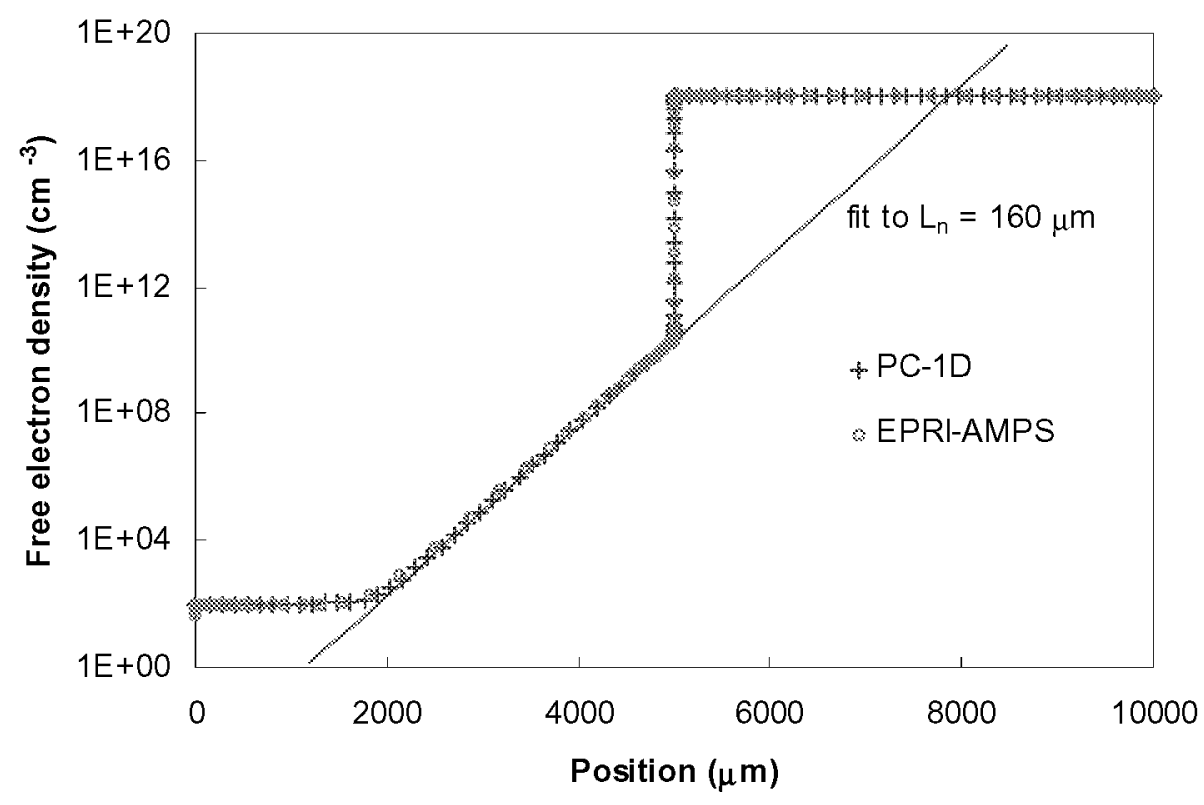

Figure 1. Simulated free electron density of a p-n junction with a forward bias of $0.5 \mathrm{~V}$.

energy in sub-band-gap region of the energy band. The DOS used in the EPRI-AMPS simulation, $n_{E}$, was $1 E 14$ $\mathrm{cm}^{-3} \mathrm{eV}^{-1}$; the cross section for the states, $\sigma_{t}$, was $1 \mathrm{E}-16 \mathrm{~cm}^{2}$. The thickness of the layers, $5,000 \mu \mathrm{m}$, was chosen to insure that all recombination occurs in the bulk of the material and not at the contacts.

The exponential decay of minority carriers in the p-type layer can be fit by an exponential with two parameters, namely, the injected excess electron minority carrier density, $\Delta n_{p}=1 E 10 \mathrm{~cm}^{-3}$ at $5,000 \mu \mathrm{m}$, and an electron diffusion length, $L_{n}=160 \mu \mathrm{m}$. The fit of the excess electron carrier density, $\delta n\left(x_{p}\right)$, to the exponential behavior in the $p$-type layer with $x_{p}<5000 \mu m$ is shown as the solid line in Figure 1 ; it was obtained using:

$$
\delta n\left(x_{p}\right)=\Delta n_{p} \exp \left[\left(x_{p}-5000 \mu m\right) / L_{n}\right] .
$$

The electron diffusion length can be calculated from the electron mobility, $\mu_{n}$, and lifetime, $\tau_{n}$, using:

$$
L_{n}=\operatorname{sqrt}\left[(k T / q) \mu_{n} \tau_{n}\right)
$$

where $\mathrm{k}$ is the Boltzman constant, $\mathrm{T}$ the temperature, $\mathrm{q}$ the electronic charge, and $\tau_{\mathrm{n}}$ the electron recombination lifetime. Substituting $\mu_{n}=1000 \mathrm{~cm}^{2} V^{-1} \mathrm{~s}^{-1}$ and $\tau_{n}=10 \mu$ s into Equation (2) yields $L_{n}=160 \mu \mathrm{m}$. Hence the lifetime model employed in the PC-1D simulation is in agreement with the analytical theory.

An equivalent DOS lifetime for comparison with the PC-1D simulator was calculated from the parameters used in the EPRI-AMPS DOS simulation using [8]:

$$
\tau_{\mathrm{n}}=\left(\mathrm{n}_{\mathrm{E}} \Delta \mathrm{E} \sigma_{\mathrm{t}} \mathrm{v}_{\mathrm{t}}\right)^{-1}
$$

where $\Delta \mathrm{E}$ is the energy width of the DOS distribution for states serving as recombination centers and $\mathrm{v}_{\mathrm{t}}$ is the carrier thermal velocity. Using $n_{E}=1 E 14 \mathrm{~cm}^{-3} \mathrm{eV}^{-1}, \Delta \mathrm{E}=0.1 \mathrm{eV}, \sigma_{\mathrm{t}}=1 \mathrm{E}-16 \mathrm{~cm}^{2}$ and $v_{\mathrm{t}}=1 \mathrm{E} 7 \mathrm{~cm} / \mathrm{s}$, yields a lifetime of $100 \mu \mathrm{s}$, which is ten times greater than the lifetime used in the PC-1D simulation. The free electron density shown in Figure 1 is in agreement with the PC-1D simulation, and the two simulations are in agreement with analytical theory for a two-sided step p-n junction. However, the discrepancy in the lifetimes must be resolved if 


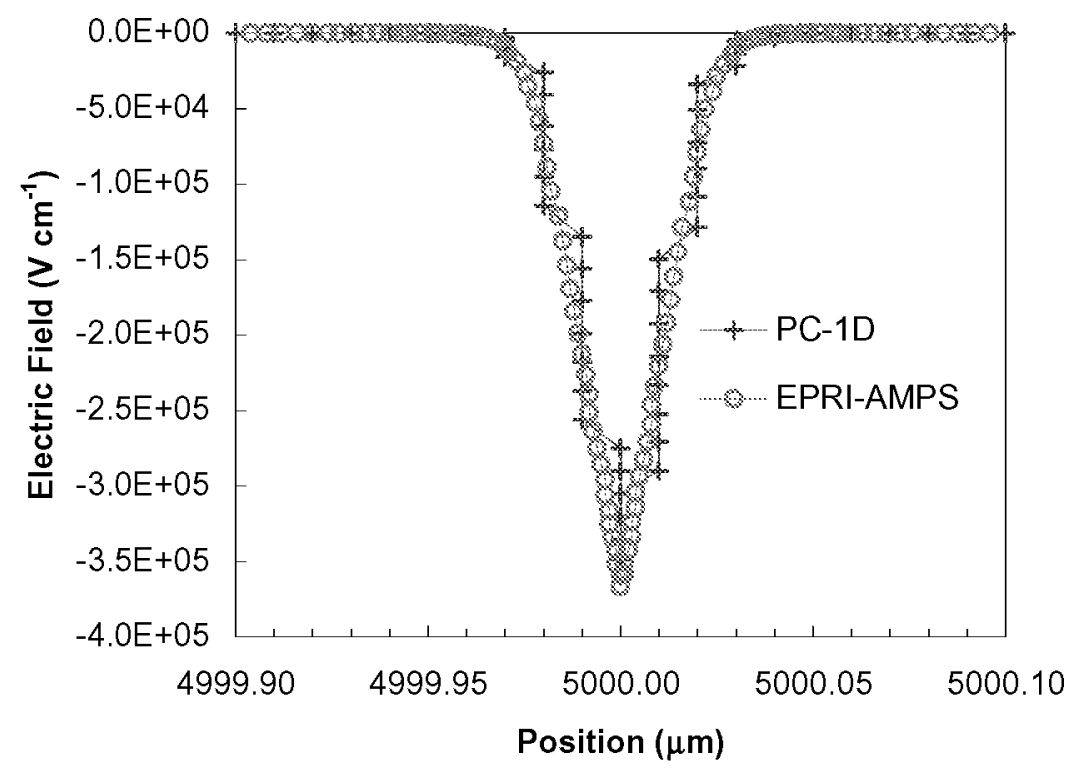

Figure 2. Simulated electric field of $p-n$ junction near the metallurgical junction with a forward bias of $0.5 \mathrm{~V}$.

one is to have confidence in applying the simulators to the analysis of devices. We will explore the discrepancy in future work.

The position dependence of the electric field was simulated with the two simulators. The results are shown in Figure 2. The electric field has the largest value at the metallurgical junction and the depletion width is about 0.05 $\mu \mathrm{m}$ for both simulations. The step like behavior in the results for the PC-1D simulation is due to rounding-off errors. The electric fields predicted by both simulators are in agreement with the analytical model.

The simulated dark J-V characteristics of the p-n junction for the EPRI-AMPS and PC-1D simulators are shown in Figure 3. Also shown is the dark J-V characteristic calculated with the analytical model:

$$
\left.J(V)=J_{0}[\exp (q V / k T)-1] \text { where } J_{0}=(k T)\left(\mu_{p} p_{n} / L_{p}+\mu_{n} n_{p} / L_{n}\right)\right] .
$$

Substituting the values for the Boltzman constant, temperature, mobilities, minority carrier densities and diffusion lengths and into Equation (4) results in the straight line shown in Figure 3. There is good agreement between the analytical model and the simulated results in the 0.2 to $0.8 \mathrm{~V}$ range of forward biases. As mentioned above, the PC-1D simulator is a transient simulator, and for this reason, current densities at low voltages with depend on the device capacitance and voltage step rate. The effect of varying the step rate of the forward-bias voltage on the dark J-V characteristics is shown in Figure 4. The current charges the junction capacitance at low biases. For these simulations, the $p-n$ junction capacitance is $0.2 \mu \mathrm{F} / \mathrm{cm}^{2}$. When the voltage is ramped at $10 \mathrm{mV} / \mathrm{s}$, which corresponds to $1 \mathrm{~s} / \mathrm{step}$, the current at voltages less than $200 \mathrm{mV}$ is $2 \mathrm{e}-9 \mathrm{~A} / \mathrm{cm}^{2}$. Reducing the step rate to 100 s/step results in the forward-biased current decreasing by an order of magnitude. A $1000 \mathrm{~s} / \mathrm{step}$ decreases the current further. The dark J-V characteristic approaches the characteristic that would be expected for the analytical model. 


\section{SINGLE-JUNCTION p-i-n SOLAR CELL MEASUREMENTS AND SIMULATIONS}

Single junction p-i-n a-Si:H solar cells were deposited on bare stainless steel substrates without the use of textured back reflectors. An n-type layer about $15 \mathrm{~nm}$ thick was deposited on the bare stainless steel substrate followed by the deposition of a high quality a-Si:H intrinsic layer about $200 \mathrm{~nm}$ thick. An $8 \mathrm{~nm}$ thick p-type layer was then deposited on the i-type layer. All a-Si:H layers were deposited using radio-frequency plasma enhanced

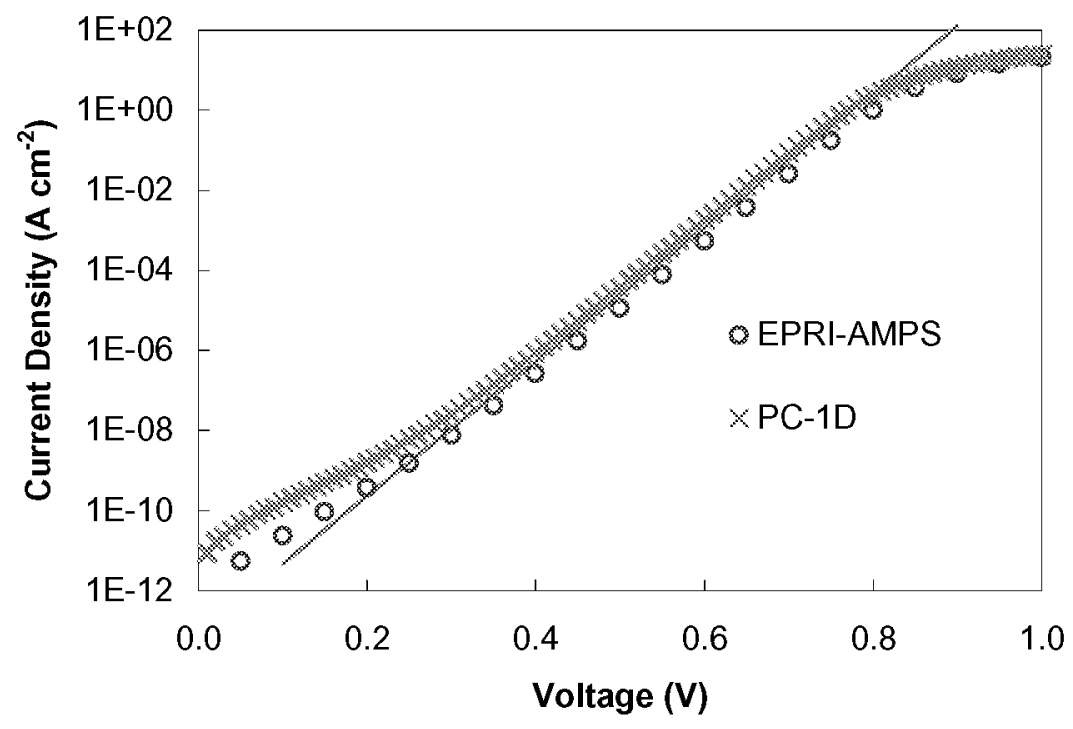

Figure 3. EPRI-AMPS and PC-1D simulated dark J-V characteristic for a two-sided-step p-n junction.

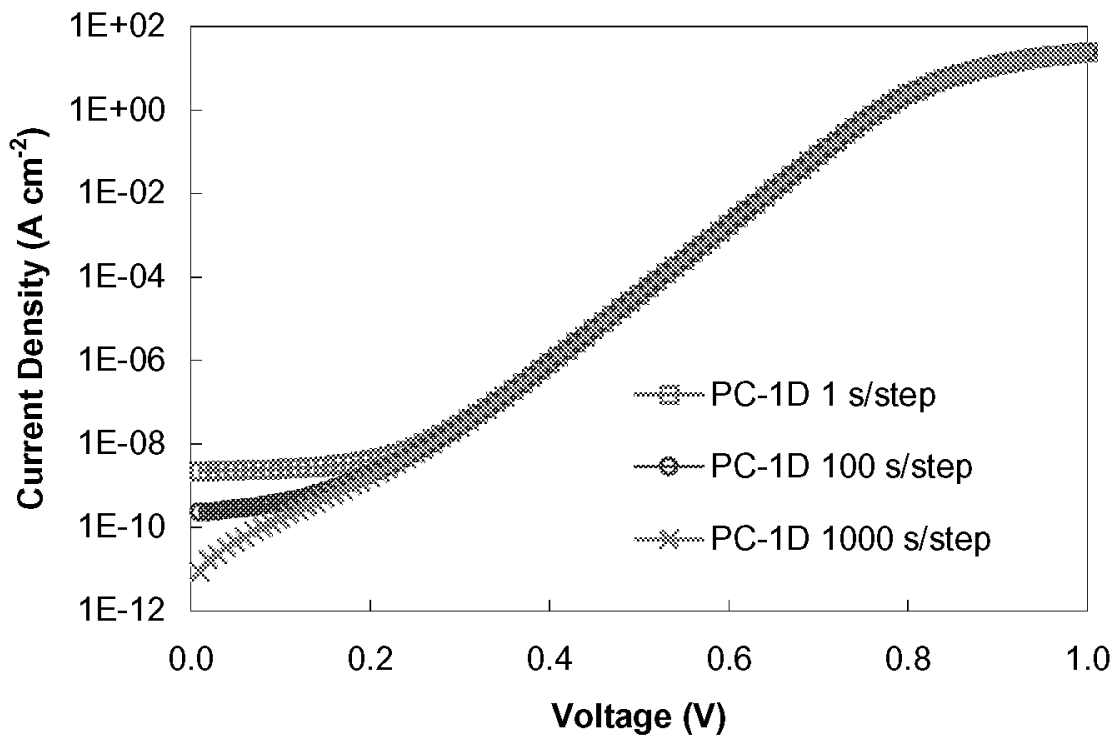

Figure 4. PC-1D simulated dark J-V characteristics for a two-sided step p-n junction with three voltage step rates. 
chemical vapor deposition. Indium tin oxide, ITO, was evaporated through a mask with an area of $0.25 \mathrm{~cm}^{2}$. The ITO serves as the top contact and as a $1 / 4$ wavelength anti-reflective coating. Finally, Au grids were evaporated through a mask on top of the ITO. Since the cells do not employ a light scattering back reflector, the initial efficiency of the devices is about $6 \%$. Initial dark J-V characteristics were measured after the virgin cell was annealed for two hours at $200^{\circ} \mathrm{C}$ in a vacuum system at about 1E-6 Torr. A Cambridge S250 MK3 scanning electron microscope was used to irradiate the single-junction $\mathrm{p}$-i-n solar cells with $40 \mathrm{keV}$ electrons. The electron current was about $0.6 \mu \mathrm{A}$ and the beam scanned over an area of about $0.55 \times 0.72 \mathrm{~cm}^{2}$. Irradiation times of five hours were necessary to obtain a fluence of about $1 \mathrm{E} 17 \mathrm{~cm}^{-2}$. Following the irradiation, dark J-V characteristics were measured again. The cells were annealed a second time. A subsequent irradiation and annealing cycle was conducted. Dark J-V characteristics were measured between each irradiation and annealing step. The dark $\mathrm{J}-\mathrm{V}$ characteristics were measured with a computer-interfaced Keithley Instruments model 236 source measurement unit that is programmed to source the bias voltage in $10 \mathrm{mV}$ increments and measure the current at 111 separate voltages in the range of 0 to $1.10 \mathrm{~V}$. The instrument and software are configured to make 320 current measurements at each source-bias voltage and calculate the average and standard deviation of the current. It took three to four minutes to measure a single $\mathrm{J}-\mathrm{V}$ characteristic. The standard deviation for each of the measurements was calculated and is greater than 100 for the measurements reported in this work. This insures that the average current is representative of the device and not a transient current due to junction capacitance.

The energy deposition of the irradiating $40 \mathrm{keV}$ electrons is assumed to be uniform in the cells studied in this work. While it is possible to use particle-solid interaction models to determine the spatial dependence of the electron-hole generation rate produced by the irradiation, our interest is in determining the role of the material properties on the dark J-V characteristics. Since the energy deposition is assumed to be uniform, simulated material properties were chosen to be uniform in the intrinsic layer with respect to position. This assumes that radiation induced defects do not migrate from the position were they are created.

The measured dark J-V characteristics of the p-i-n solar cell before irradiation are shown by the dots in Figure 5. The dark J-V characteristic measured after annealing at $200^{\circ} \mathrm{C}$ for 2 hours is labeled a11. A second J-V characteristic, a12, was measured immediately following the a11. The currents for both the a11 and the a12 measurements are linear on the semi-log graph over a range of $1 \mathrm{E}-10$ to $1 \mathrm{E}-4 \mathrm{~A} / \mathrm{cm}^{2}$. The data show that the measurement of a11 influenced the measurement of a12. Subsequent measurements of the dark J-V characteristics are similar to the a12 measurements, thereby showing saturation of the effect that produced the a12 dark J-V characteristic. Hence, the cell was annealed before each irradiation. EPRI-AMPS simulations were carried out with various material parameters in an effort to obtain a good fit between the measured simulated dark $\mathrm{J}-\mathrm{V}$ characteristics. The most influential parameter that affected the dark J-V characteristics was found to be the DOS near the center of the band gap. A good fit was obtained with a DOS made up of a discrete energy level with an energy width of $0.1 \mathrm{eV}$ and located at the center of the band gap. Equal numbers of acceptor and donor like states were used for the discrete energy states. The results of simulations with various densities of discrete energy states are plotted in Figure 5 as solid lines. The density of discrete energy states was increased from 0 to $1 \mathrm{E} 17 \mathrm{~cm}^{-3} \mathrm{eV}^{-1}$. The simulated results can be fitted with an expression of the form:

$$
J(V)=J_{0}[\exp (q V / n k T)-1]
$$

where $\mathrm{n}$ is the material quality factor. As the density of discrete energy states at the center of the band gap is increased, $J_{0}$ and $n$ increase. The curve labeled 0 corresponds to a zero discrete energy state density at the center of the sub-band-gap. The recombination for a simulation with no discrete energy states is dominated by recombination from the conduction band-tail states to the valence band-tail states. Figure 5 shows that the $J_{0}$ and $\mathrm{n}$ terms in Equation (5) for the curve labeled 0 are significantly less than for the measured a11 data. The curve labeled 5E15 represents a simulation with a discrete energy state density of $5 \mathrm{E} 15 \mathrm{~cm}^{-3} \mathrm{eV}^{-1}$ at the center of the band gap. The $J_{0}$ and $n$ terms for this simulation are in agreement with the a11 data over 6 orders of magnitude.

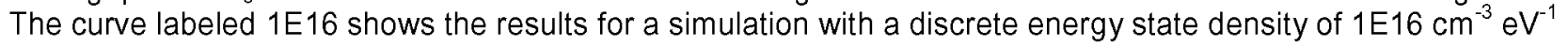
located at the center of the band gap. This simulation is in agreement with the a12 data over 6 orders of magnitude as well. The deviation in linearity between the simulated and measured dark $\mathrm{J}$-V characteristics above $0.8 \mathrm{~V}$ is believed to be due to series resistance in the measured cells. The series resistance results from factors external to the p-i-n structure, such as contact resistance and resistance in the ITO layer. Future simulations including these factors will be carried out to obtain a better fit of the dark J-V characteristics above $0.8 \mathrm{~V}$. 


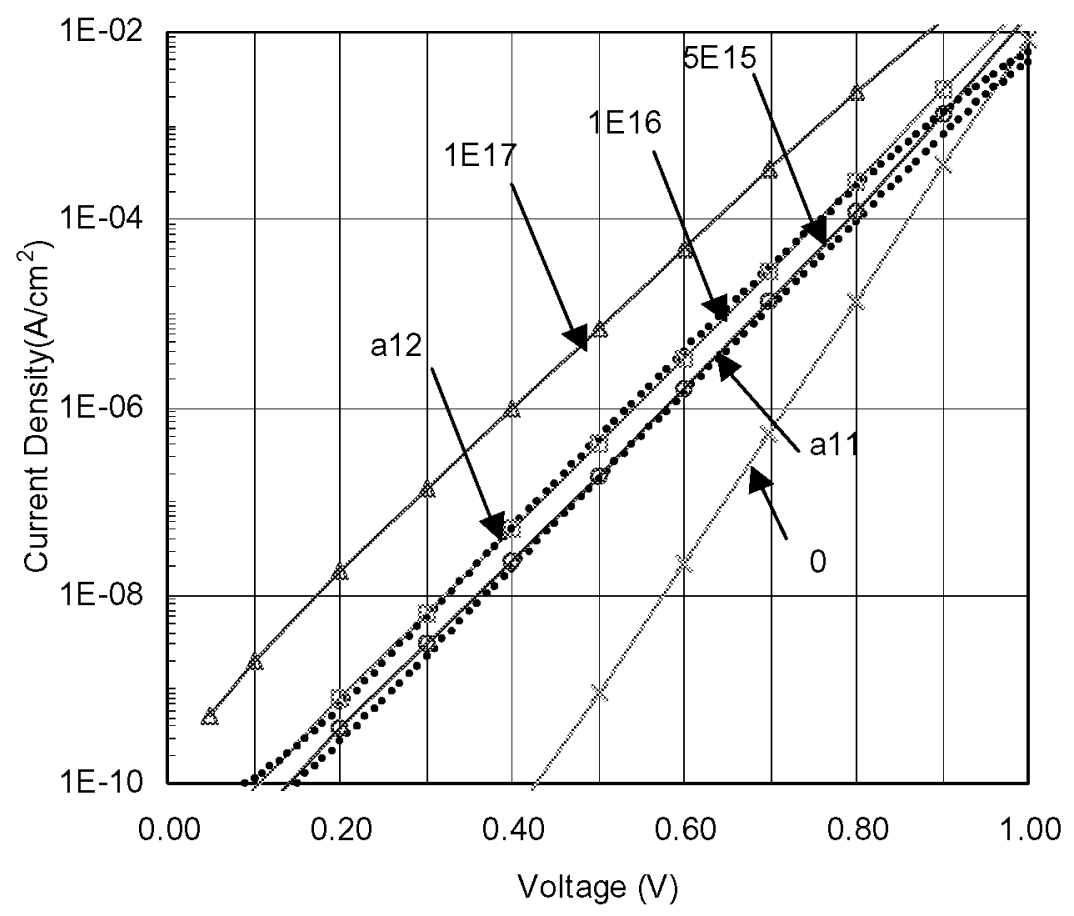

Figure 5. Measured and EPRI-AMPS simulated dark J-V characteristics for an a-Si:H $p-i-n$ solar cell before irradiation.

Figure 5 suggests that the difference between the a11 and a12 J-V characteristics is due to an increase in the sub-band-gap energy state density from $5 \mathrm{E} 15 \mathrm{~cm}^{-3} \mathrm{eV}^{-1}$ to $1 \mathrm{E} 16 \mathrm{~cm}^{-3} \mathrm{eV}^{-1}$.

A dark J-V characteristic of a p-i-n solar cell after irradiation with $40 \mathrm{keV}$ electrons to a fluence of $1 \mathrm{E} 17 \mathrm{~cm}^{-2}$ is shown in Figure 6 by the curve made up of dots and labeled $r 1$. For comparison purposes, the measured dark $\mathrm{J}$ $V$ characteristic before irradiation is also shown by the curve made up of dots and labeled a1. Simulations were carried out with the EPRI-AMPS simulator using a discrete energy state located at the center of the band gap with densities of $7 \mathrm{E} 17$ and $8 \mathrm{E} 17 \mathrm{~cm}^{-3} \mathrm{eV}^{-1}$. The simulated results are shown by the solid curves in Figure 6 that are labeled 7E17 and 8E17. Our previous work shows that there are three current mechanisms that contribute to the dark J-V characteristic of irradiated a-Si:H solar cells, namely, injection, shunt and CV mechanisms [9]. The current injection mechanism is evident in the $r 1$ curve since the current increases exponentially with forward-bias voltage above 0.7 Volts. Below $0.7 \mathrm{~V}$ the shunt and $\mathrm{CV}^{\mathrm{m}}$ mechanisms appear to contribute to the current. The $\mathrm{r} 1$ dark $\mathrm{J}-\mathrm{V}$ characteristic has higher $\mathrm{J}_{0}$ and $\mathrm{n}$ values than the a1 characteristic. The simulated results suggest that a good fit to the irradiated cell J-V characteristic above $0.7 \mathrm{~V}$ may be obtained with a discrete energy state density of about $7.5 \mathrm{E} 17 \mathrm{~cm}^{-3} \mathrm{eV}^{-1}$.

The results of the EPRI-AMPS dark J-V simulations in Figure 5 show that the cell current density for a given forward-bias voltage increases with increasing discrete energy state density up to $1 \mathrm{E} 17 \mathrm{~cm}^{-3} \mathrm{eV}^{-1}$. However, Figure 6 shows that increasing the discrete energy state density further to $7 \mathrm{E} 17$ and $8 \mathrm{E} 17 \mathrm{~cm}^{-3} \mathrm{eV}^{-1}$ results in a decrease in the current density at a given voltage. Note that the measured J-V characteristic above $0.7 \mathrm{~V}$ shown in Figure 6 for the irradiated cell has a lower current density than that of the annealed cell for the same forwardbias voltages. Hence, in the forward-bias voltage range where current the injection mechanism dominates, the effect of irradiation on the cell is to produce a behavior in the measured J-V characteristic that is the same as increasing the discrete energy state density in the simulated dark J-V characteristics. It is plausible to assume that $40 \mathrm{keV}$ electron irradiation produces defect states near the center of the band gap that serve as 


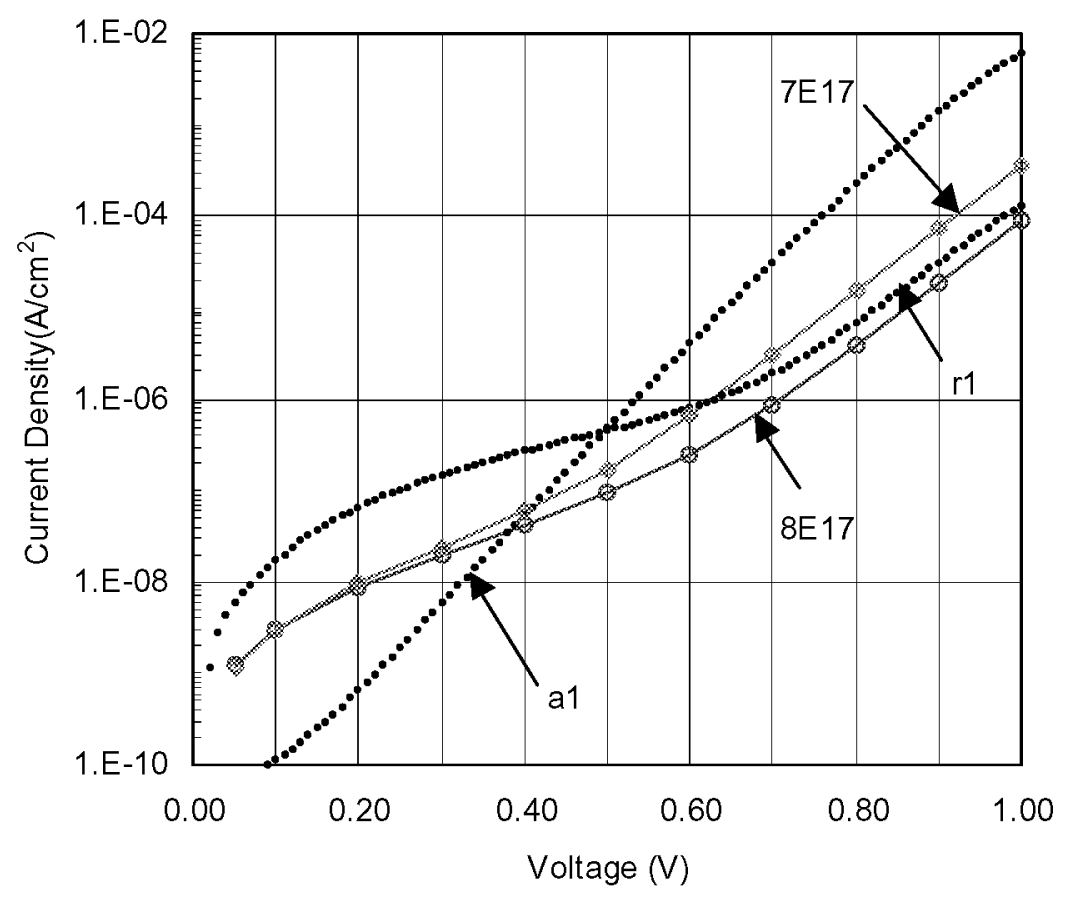

Figure 6. Measured and EPRI-AMPS simulated dark J-V characteristics for an a-Si:H p-i-n solar cell before and after irradiation with $40 \mathrm{keV}$ electrons.

recombination centers. The behavior can also be explained by noting the effect of increasing the discrete energy state density, whether by simulation or irradiation, is to increase the values of $J_{0}$ and $n$ in Equation (5). The simulated dark $\mathrm{J}-\mathrm{V}$ characteristics plotted in Figure 6 show that the higher discrete energy state densities result in a non-linear current density on the log scale below $0.7 \mathrm{~V}$. The same effect exists in the measured dark J-V characteristic of the irradiated cell, but it is much more pronounced; the magnitude of the current density is about an order of magnitude larger for the measured cells. In our earlier work that employed parametric fitting, we associated this effect with a shunting mechanism and a current mechanism that results in a term of the form $\mathrm{CV}^{\mathrm{m}}$. The EPRI-AMPS simulation studies provide an understanding of the dark J-V characteristic in the forward-bias region where the current injection mechanism dominates. Studies need to be carried out to understand the physical bases of the current mechanisms at forward biases below $0.7 \mathrm{~V}$.

\section{CONCLUSIONS}

Studies have been carried out to correlate the effect of $40 \mathrm{keV}$ electron irradiation on dark J-V characteristics. EPRI-AMPS and PC-1D simulators were utilized to characterize a two-sided step p-n junction before selecting a simulator for investigating a $\mathrm{p}-\mathrm{i}-\mathrm{n}$ solar cell structure. The two simulators produced dark J-V characteristics that were in agreement each other and an analytical model, but the lifetimes used in the two simulators differ by a factor of ten. The EPRI-AMPS simulator was used to simulate dark J-V characteristics of a $p$-i-n solar cell. In the forward-bias voltage range above $0.7 \mathrm{~V}$, where the current injection mechanism dominates, the effect of irradiation on the cell is to produce a behavior in the measured $\mathrm{J}-\mathrm{V}$ characteristic that is the same as increasing the discrete energy state density in the simulated dark J-V characteristics. It is plausible to assume that $40 \mathrm{keV}$ electron irradiation produces defect states near the center of the band gap and that the states serve as recombination centers. The EPRI-AMPS simulation studies provide an understanding of the dark J-V characteristic when current injection dominates. Studies need to be carried out to understand the physical bases of the current mechanisms at forward biases below $0.7 \mathrm{~V}$. 


\section{REFERENCES}

1. Ultra-light Amorphous Silicon Alloy Photovoltaic Modules for Space Applications, J. J. Hanak, E. Chen, C. Fulton, A. Myatt and J. R. Woodyard, Space Photovoltaic Research and Technology Conference, 1986, NASA Conference Publication 2475, page 99.

2. Investigation of Current Mechanisms in a-Si:H Alloy Cells, Kenneth R. Lord II, Fazal UrRahman Syed, Michael R. Walters and James R. Woodyard, First World Conference on Photovoltaic Energy Conversion, Twenty Fourth IEEE Photovoltaic Specialists Conference-1994, page 993.

3. James R. Woodyard and Geoffry Landis, "Radiation resistance of thin-film solar cells for space photovoltaic power," Solar Cells, 31, pp. 297-329, 1991.

4. Thin Film Development Workshop Report, J. R. Woodyard, Eleventh Space Photovoltaic Research and Technology Conference-1991, NASA Conference Publication 3121, 1991, page 48-1.

5. Solid State Devices, Ben G. Streetman and Sanjay Banerjee, Prentice Hall, 2000, pages 157-185.

6. Dr. Stephen Fonash and collaborators at The Pennsylvania State University developed EPRI-AMPS; information may be obtained at http://www.psu.edu/dept/AMPS/.

7. Dr. Paul Basore and collaborators developed PC-1D; information may be obtained at www.pv.unsw.edu.au/pc1d/.

8. Physics and Technology of Semiconductor Devices, A. S. Grove, John Wiley and Sons, 1967, page 134.

9. Qianghua Wang, Kenneth Lord, and James R. Woodyard, "Effects of $40 \mathrm{keV}$ electron irradiation on dark I-V characteristics of single-junction a-Si:H solar cells," Proceedings of the $28^{\text {th }}$ Photovoltaics Specialists Conference, pp. 1057-1060. 